\title{
Risk of Cardiovascular Conditions in Patients with Chronic Hypoparathyroidism: A Retrospective Cohort Study
}

\author{
Elvira O. Gosmanova (1) - Kristina Chen • Markus Ketteler • \\ Lars Rejnmark · Fan Mu • Elyse Swallow · Allison Briggs • \\ Nicole Sherry $\cdot$ Sanjiv Kaul
}

Received: March 19, 2021 / Accepted: May 10, 2021 / Published online: June 24, 2021

(C) The Author(s) 2021

\section{ABSTRACT}

Introduction: In patients with chronic hypoparathyroidism disordered calcium homeostasis has been associated with risk of cardiovascular diseases, including cardiomyopathy,

Supplementary Information The online version contains supplementary material available at https:// doi.org/10.1007/s12325-021-01787-7.

E. O. Gosmanova ( $\square)$

Division of Nephrology and Hypertension, Albany

Medical College, Albany, NY, USA

e-mail: Elvira.Gosmanova@va.gov

K. Chen · N. Sherry

Shire Human Genetic Therapies, Inc., a Takeda

Company, Lexington, MA, USA

M. Ketteler

Department of General Internal Medicine and

Nephrology, Robert-Bosch-Krankenhaus, Stuttgart,

Germany

M. Ketteler

University of Split School of Medicine, Split, Croatia

L. Rejnmark

Department of Endocrinology and Internal

Medicine, Aarhus University Hospital, Aarhus

University, Aarhus, Denmark

F. Mu · E. Swallow · A. Briggs

Analysis Group, Inc., Boston, MA, USA

S. Kaul

Knight Cardiovascular Institute, Oregon Health and

Science University, Portland, OR, USA congestive heart failure, and arrhythmia; however, larger-scale studies are needed to examine these risks. This study evaluated the risk of cardiovascular conditions among patients with chronic hypoparathyroidism.

Methods: Adults with and without chronic hypoparathyroidism were selected from a medical insurance claims database in the USA from January 2007 to June 2017, and were followed for up to 5 years. Associations between chronic hypoparathyroidism and incident atrial fibrillation (AF), tachyarrhythmia, myocardial infarction (MI), coronary artery disease (CAD), heart failure (HF), stroke, cerebrovascular disease, peripheral vascular disease (PVD), and a combined cardiovascular endpoint of cerebrovascular disease, CAD, HF, and PVD were compared between cohorts using Kaplan-Meier analyses and unadjusted and adjusted Cox proportional hazards models.

Results: In 8097 patients with chronic hypoparathyroidism compared with 40,485 patients without, respectively, mean \pm SD ages were $58.6 \pm 16.3$ and $47.3 \pm 18.0$ years, $76.2 \%$ and $54.4 \%$ were female, and $19.4 \%$ and $9.5 \%$ had the combination of cardiovascular findings at baseline. In adjusted analyses, patients with chronic hypoparathyroidism had significantly higher risk (adjusted hazard ratio and 95\% confidence interval) of incident AF (1.72; $1.51-1.97)$, tachyarrhythmia $(1.68 ; 1.32-2.14)$, MI (1.18; 1.01-1.38), CAD (1.39; 1.26-1.54), HF $(1.64 ; 1.46-1.84)$, stroke $(1.45 ; 1.31-1.62)$, 
cerebrovascular disease $(1.48 ; 1.34-1.62)$, PVD (1.66; 1.51-1.81), and combined cardiovascular endpoint (1.63; 1.52-1.75), all $P<0.001$ except $P=0.036$ for MI, compared with patients without chronic hypoparathyroidism.

Conclusions: This large retrospective cohort study showed that chronic hypoparathyroidism was associated with increased risk of incident cardiovascular conditions and arrhythmias. Results should be evaluated in light of limitations inherent to claims database analyses. Further studies are warranted to investigate reasons for these risks and to develop strategies for reducing cardiovascular conditions in patients with chronic hypoparathyroidism.

Keywords: Arrhythmia; Cardiovascular disease; Chronic hypoparathyroidism; Retrospective cohort study

\section{Key Summary Points}

Why carry out this study?

Chronic hypoparathyroidism is a rare endocrine disorder characterized by insufficient parathyroid hormone, hypocalcemia, and hyperphosphatemia.

Studies in small cohorts of patients $(\leq 700)$ with hypoparathyroidism showed that hypocalcemia was associated with poor cardiovascular outcomes, including cardiomyopathy, congestive heart failure, and arrhythmia.

This retrospective cohort study examined risks of development of cardiovascular conditions in 8097 patients with chronic hypoparathyroidism compared with 40,485 patients without hypoparathyroidism.

\section{What was learned from the study?}

Patients with chronic hypoparathyroidism had significantly higher risk of incident cardiovascular conditions compared with those without hypoparathyroidism.
Further studies are necessary to understand the underlying mechanisms for the associations between cardiovascular conditions and chronic hypoparathyroidism.

\section{DIGITAL FEATURES}

This article is published with digital features, including a summary slide, to facilitate understanding of the article. To view digital features for this article go to https://doi.org/10.6084/ m9.figshare.14561874.

\section{INTRODUCTION}

Chronic hypoparathyroidism is a rare endocrine disorder characterized by hypocalcemia and hyperphosphatemia resulting from insufficient production of parathyroid hormone (PTH) $[1,2]$. Per 100,000 individuals, hypoparathyroidism affects an estimated 37 individuals in the USA [1]. The role of PTH is to regulate homeostasis of calcium and phosphate; PTH deficiency leads to diminished calcium reabsorption and phosphate excretion in the kidneys, diminished calcium and phosphate absorption in the gastrointestinal tract, and decreased bone remodeling, resulting in significant hypocalcemia and mild hyperphosphatemia [1,3]. In patients with hypoparathyroidism, disordered calcium homeostasis (hypocalcemia or a history of at least four episodes of hypercalcemia) has been associated with poor cardiovascular (CV) outcomes including cardiomyopathy, congestive heart failure, and arrhythmia [3-7]. Moreover, elevated calcium phosphate product is associated with vascular and with valvular calcifications that are associated with all-cause and CV mortality [8-10].

Studies investigating the association between hypoparathyroidism and risk of $\mathrm{CV}$ events have yielded mixed results. A singlecenter study using a Danish registry reported 
that in patients with postsurgical chronic hypoparathyroidism, there was not an increased risk of CV disease compared with ageand gender-matched controls [11], whereas patients with nonsurgical hypoparathyroidism and those with disturbances in calcium homeostasis were at increased risk of $\mathrm{CV}$ diseases $[7,12,13]$. Similarly, a Scottish populationbased study found increased risk versus age- and gender-matched controls [14]. Moreover, the potential contribution of conventional therapy for chronic hypoparathyroidism, which consists of oral administration of calcium and active vitamin $\mathrm{D}$ supplementation $[2,15]$, toward $\mathrm{CV}$ risk is not known.

As current knowledge on CV risk in individuals diagnosed with chronic hypoparathyroidism is informed mainly by small cohort studies, there is a need for large-scale studies to better our understanding of hypoparathyroidism and CV risk. Accordingly, we performed a retrospective cohort study to examine the association between chronic hypoparathyroidism and risk of various $\mathrm{CV}$ conditions using a large managed care commercial claims database available in the USA.

\section{METHODS}

\section{Data Source and Study Design}

This retrospective study extracted data from a large managed care claims database in the USA covering both commercial (employer database) and Medicare Advantage beneficiaries and consisting of more than 14 million members per year between January 2007 and June 2017. Claims are sourced from a single national health insurance carrier. Because this study used de-identified licensed data from a Health Insurance Portability and Accountability Act (HIPAA)-compliant managed care database, ethics committee approval and informed consent were not required.

Patients were grouped into two cohorts: those with chronic hypoparathyroidism and those without chronic hypoparathyroidism. Selection criteria are shown in Fig. 1. All eligible patients were required to be aged at least
18 years on the index date, have at least 6 months of continuous eligibility before the index date, and have no evidence of treatment with recombinant human PTH therapies rhPTH(1-84) or rhPTH(1-34). For patients with chronic hypoparathyroidism, the index date was defined as the date of the first repeat diagnosis of hypoparathyroidism at least 6 months after the initial diagnosis. For patients without hypoparathyroidism, the index date was defined as the date of an eligible, randomly selected claim. The final group of control patients was obtained by random selection at a ratio of 5:1 (without chronic hypoparathyroidism: with chronic hypoparathyroidism). For both patient cohorts, the baseline period was defined as the 6 months before the index date. Patients were followed for up to 5 years after the index date or until the end of continuous eligibility (i.e., uninterrupted coverage by insurance provider), whichever occurred first.

\section{Outcome Measures}

International Statistical Classification of Diseases and Related Health Problems, 9th (ICD-9) or 10th (ICD-10) Revision Clinical Modification diagnosis or Procedure Coding System codes, Healthcare Common Procedure Coding System codes, or Current Procedural Terminology codes were used to identify the first diagnosis of each respective $\mathrm{CV}$ outcome during the study period (Supplementary Table 1). The following CV conditions were assessed: atrial fibrillation (AF), cerebrovascular disease, coronary artery disease (CAD), heart failure (HF), myocardial infarction (MI), peripheral vascular disease (PVD), stroke, and tachyarrhythmia. A combined CV endpoint was defined using the diagnosis and procedural codes for one or more of the following $\mathrm{CV}$ conditions: cerebrovascular disease, CAD, HF, and PVD.

\section{Statistical Analyses}

Baseline demographic and clinical characteristics of the patients with and without chronic hypoparathyroidism were evaluated using descriptive statistics (mean, standard deviation 
(a) Patients With Hypoparathyroidism

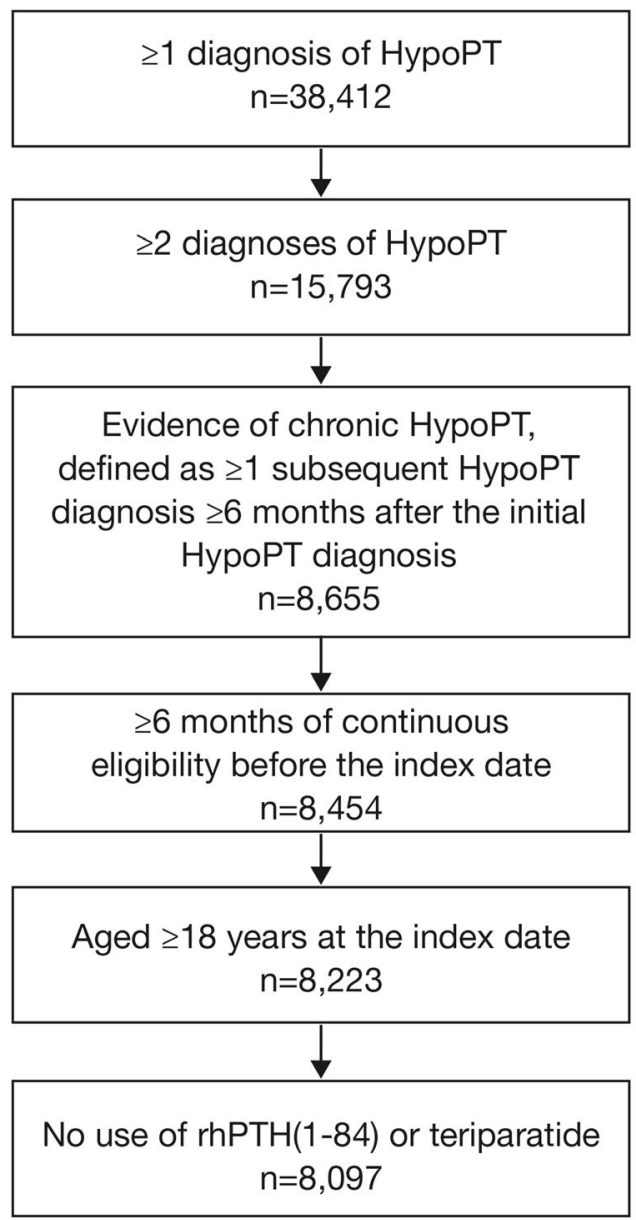

(b) Patients Without Hypoparathyroidism



Fig. 1 Sample selection of patients (a) with chronic hypoparathyroidism compared with (b) those without hypoparathyroidism. HypoPT hypoparathyroidism, $\operatorname{rhPTH}(1-84)$ recombinant human parathyroid hormone (1-84)

[SD], percentage). Patient characteristics between cohorts were compared for continuous and dichotomous variables using Wilcoxon rank sum and chi-square tests, respectively.

For each analysis, the risk of the respective $\mathrm{CV}$ condition was assessed among patients free of that $\mathrm{CV}$ condition during the baseline period. Individual $\mathrm{CV}$ outcomes and the combined $\mathrm{CV}$ endpoint were compared using Kaplan-Meier analyses with log-rank tests. Between-cohort comparisons were made using unadjusted and adjusted Cox proportional hazards models. Multivariable Cox models adjusted for demographic characteristics (age, sex, race, US region, and index year) and outcome-relevant clinical characteristics (other comorbid CV conditions, chronic kidney disease [CKD], hyperlipidemia, hypertension, diabetes, and use of CV medications) at baseline. Medications with potential $\mathrm{CV}$ influence were defined by generic product identifier or Healthcare Common Procedure Coding System codes for beta blockers, statins, renin-angiotensin-aldosterone system (RAAS) blockers (aldosterone receptor antagonists, angiotensin-converting enzyme inhibitors, angiotensin receptor blockers, and direct renin inhibitors), calcium channel blockers (CCBs), or oral thiazide diuretics (Supplementary Table 2). CKD was defined by diagnosis codes. All 
statistical analyses were performed using SAS Enterprise Guide 7.1 (SAS Institute, Inc., Cary, $\mathrm{NC}$ ).

\section{RESULTS}

A total of 8097 patients with chronic hypoparathyroidism and 40,485 patients without chronic hypoparathyroidism were included in the analysis (Fig. 1). Baseline characteristics for the two study cohorts are shown in Table 1. The mean \pm SD age of patients in the chronic hypoparathyroidism cohort was approximately 10 years older than patients in the cohort without chronic hypoparathyroidism $(58.6 \pm 16.3$ vs $47.3 \pm 18.0$ years; $P<0.001)$, and a larger proportion of patients were female (76.2\% vs $54.4 \% ; P<0.001)$.

At baseline, a significantly higher proportion of patients with chronic hypoparathyroidism had $\mathrm{CV}$ conditions that comprised the combined CV endpoint compared with patients without chronic hypoparathyroidism $(19.4 \%$ vs 9.5\%). Accordingly, more patients with chronic hypoparathyroidism had individual CV conditions compared with patients who did not have hypoparathyroidism: AF (6.0\% vs $2.7 \%)$, tachyarrhythmia ( $0.7 \%$ vs $0.4 \%)$, MI ( $1.9 \%$ vs $1.3 \%)$, CAD (9.6\% vs $5.3 \%)$, HF (5.9\% vs $2.4 \%)$, stroke ( $4.6 \%$ vs $2.4 \%)$, cerebrovascular disease $(6.0 \%$ vs $3.0 \%)$, and PVD (7.4\% vs $2.8 \%$; all $P<0.001)$. Consequently, $53.0 \%$ of patients with chronic hypoparathyroidism were receiving medications with potential CV influence compared with $30.3 \%$ of patients without chronic hypoparathyroidism. Other comorbidities such as CKD stages 3 and 4, hypertension, and diabetes (types 1 and 2) were also more prevalent in patients with chronic hypoparathyroidism than those without chronic hypoparathyroidism (all $P<0.001$; Table 1).

In unadjusted Kaplan-Meier analyses, patients with chronic hypoparathyroidism had a significantly increased risk of a new occurrence of the combined $\mathrm{CV}$ endpoint, $\mathrm{MI}$, stroke (all $P<0.001$; Fig. 2), and other individual $\mathrm{CV}$ conditions (all $P<0.001$; Supplemental Fig. 1) compared with patients without chronic hypoparathyroidism. Even after adjustment for differences in demographic and clinical baseline characteristics between the two cohorts, there remained a significantly increased risk for patients with chronic hypoparathyroidism across all eight individual $\mathrm{CV}$ outcomes (AF, tachyarrhythmia, MI, CAD, HF, stroke, cerebrovascular disease, and PVD) and the combined $\mathrm{CV}$ endpoint (all $P<0.001$ except $P=0.036$ for MI) compared with those without chronic hypoparathyroidism (Table 2).

\section{DISCUSSION}

To our knowledge, this is the first large study demonstrating that patients with chronic hypoparathyroidism have a greater risk of $\mathrm{CV}$ conditions compared with patients without hypoparathyroidism. Our goal was to evaluate the impact of hypoparathyroidism on individual outcomes as well as a composite outcome representing the combined incremental burden of these conditions. After adjusting for potential confounders, the greater risk of all individual and composite $\mathrm{CV}$ outcomes in patients with chronic hypoparathyroidism compared with patients without the disease remained significant. Consistent with other studies [16], we evaluated CV endpoints including stroke and MI. Because stroke and MI are severe and acute events, all patients are equally and highly likely to seek treatment, limiting the risk of detection bias.

Previous research focusing on the relationship between hypoparathyroidism and CV events generally examined small numbers of patients outside the USA. A study comparing 180 Danish patients with nonsurgical hypoparathyroidism to gender- and agematched controls found that nonsurgical hypoparathyroidism was significantly associated with increased risk of CV disease, including ischemic heart disease [12]. In the Danish study, risks of stroke and arrhythmia were higher for patients with nonsurgical hypoparathyroidism compared with controls, and neither risk of MI nor cardiac arrest appeared to differ between cohorts [12]. A further study in Denmark showed that longer duration of hypoparathyroidism and low time-weighted serum calcium 
Table 1 Baseline characteristics during the 6 months before the index date among patients with hypoparathyroidism compared with those without a diagnosis of hypoparathyroidism

\begin{tabular}{|c|c|c|c|}
\hline Characteristic & Hypoparathyroidism & $\begin{array}{l}\text { Without } \\
\text { hypoparathyroidism }\end{array}$ & $P$ value \\
\hline Age at index date ${ }^{a}$, mean $\pm S D$, year & $58.6 \pm 16.3$ & $47.3 \pm 18.0$ & $<0.001$ \\
\hline Female, \% & 76.2 & 54.4 & $<0.001$ \\
\hline \multicolumn{4}{|l|}{ Race, \% } \\
\hline White/Caucasian & 64.7 & 63.1 & 0.006 \\
\hline Black or African American & 8.8 & 8.5 & 0.482 \\
\hline Hispanic or Latino & 10.3 & 9.8 & 0.145 \\
\hline Asian/Pacific Islander & 3.0 & 3.8 & $<0.001$ \\
\hline \multicolumn{4}{|l|}{ CV conditions, $\%$} \\
\hline $\mathrm{AF}$ & 6.0 & 2.7 & $<0.001$ \\
\hline Cerebrovascular disease & 6.0 & 3.0 & $<0.001$ \\
\hline CAD & 9.6 & 5.3 & $<0.001$ \\
\hline MI & 1.9 & 1.3 & $<0.001$ \\
\hline $\mathrm{HF}$ & 5.9 & 2.4 & $<0.001$ \\
\hline Hyperlipidemia & 37.5 & 22.8 & $<0.001$ \\
\hline PVD & 7.4 & 2.8 & $<0.001$ \\
\hline Tachyarrhythmia & 0.7 & 0.4 & $<0.001$ \\
\hline Combination of $\mathrm{CV}$ findings ${ }^{\mathrm{b}}$ & 19.4 & 9.5 & $<0.001$ \\
\hline Stroke & 4.6 & 2.4 & $<0.001$ \\
\hline Use of any medication with potential CV influence, \% & 53.0 & 30.3 & $<0.001$ \\
\hline Beta blockers & 23.0 & 10.9 & $<0.001$ \\
\hline $\mathrm{CCBs}$ & 16.5 & 7.9 & $<0.001$ \\
\hline Oral thiazide diuretics & 17.1 & 9.9 & $<0.001$ \\
\hline RAAS blockers & 23.7 & 13.8 & $<0.001$ \\
\hline Statins & 28.5 & 16.3 & $<0.001$ \\
\hline \multicolumn{4}{|l|}{ Use of medications with potential renal influence, $\%$} \\
\hline NSAIDs, PPIs, and cimetidine & 25.9 & 17.2 & $<0.001$ \\
\hline ACE inhibitors, ARBs, and diuretics & 23.5 & 13.8 & $<0.001$ \\
\hline
\end{tabular}

Other comorbidities, \%

$\mathrm{CKD}^{\mathrm{c}}$

Stage 3 
Table 1 continued

\begin{tabular}{|c|c|c|c|}
\hline Characteristic & Hypoparathyroidism & $\begin{array}{l}\text { Without } \\
\text { hypoparathyroidism }\end{array}$ & $P$ value \\
\hline ESKD & 2.6 & 0.4 & $<0.001$ \\
\hline Hypertension & 43.7 & 25.2 & $<0.001$ \\
\hline Type 1 diabetes & 2.8 & 1.1 & $<0.001$ \\
\hline Type 2 diabetes & 20.6 & 10.8 & $<0.001$ \\
\hline \multicolumn{4}{|c|}{$\begin{array}{l}A C E \text { angiotensin-converting enzyme, } A F \text { atrial fibrillation, } A R B \text { angiotensin II receptor blocker, } C A D \text { coronary artery } \\
\text { disease, } C C B \text { calcium channel blocker, } C K D \text { chronic kidney disease, } C V \text { cardiovascular, } e G F R \text { estimated glomerular fil- } \\
\text { tration rate, } E S K D \text { end-stage kidney disease, } H F \text { heart failure, } H y p o P T \text { hypoparathyroidism, } M I \text { myocardial infarction, } \\
\text { NSAID nonsteroidal anti-inflammatory drug, PPI proton pump inhibitor, } P V D \text { peripheral vascular disease, } \\
R A A S \text { renin-angiotensin-aldosterone system, } S D \text { standard deviation } \\
\text { a Index date is the date of the first hypoparathyroidism diagnosis at least } 6 \text { months after the initial hypoparathyroidism } \\
\text { diagnosis or, for the cohort without hypoparathyroidism, the date of an eligible, randomly selected claim } \\
\text { b Including cerebrovascular disease, CAD, HF, and PVD } \\
\text { c Defined by diagnosis codes }\end{array}$} \\
\hline
\end{tabular}

levels were associated with increased risk of $\mathrm{CV}$ diseases [7]. A population-based study $(N=280)$ conducted in the UK also found that patients with hypoparathyroidism were at greater risk of developing CV disease and cerebrovascular disease compared with those in gender- and agematched controls [17]. In the current study, patients with chronic hypoparathyroidism already had a higher CV burden at baseline, as demonstrated by the combination of CV findings, and the difference was further substantiated at follow-up. The present study confirms and expands on the associations found between hypoparathyroidism and multiple CV conditions among a large-scale cohort of nationally representative patients in the USA.

Although a few previous studies and the current study have identified an increased risk of CV conditions in patients with hypoparathyroidism, little is known about the mechanism(s) driving this association. Oral administration of calcium and active vitamin D as part of conventional therapy for chronic hypoparathyroidism could be a potential risk factor for increased $\mathrm{CV}$ disease. Oral calcium supplements and active vitamin D are prescribed to maintain serum calcium concentrations in the lower part of the normal range $[5,15,18,19]$. In recent years, a number of studies in broadly defined patient populations that were not limited to hypoparathyroidism have suggested that calcium supplementation may increase the risk of a wide array of $\mathrm{CV}$ events [20-22]. Results from prospective clinical studies have supported this hypothesized link between calcium supplementation and $\mathrm{CV}$ events, including vascular calcification, MI, stroke, coronary heart disease, ischemic heart disease, and CV mortality [20, 23, 24]. In patients with hyperparathyroidism, increased serum calcium level was associated with arterial stiffness that may predispose to cardiovascular disease [25]. In addition, carotid calcifications were likely more prevalent in patients with hypoparathyroidism treated with calcium supplements and active vitamin $\mathrm{D}$ than in controls, but the small sample size precluded making any firm conclusions [26]. However, a meta-analysis of randomized controlled trials in elderly women did not find an increased risk of coronary heart disease, all-cause mortality, or MI with calcium supplementation, with or without native vitamin D [27]. Fibroblast growth factor 23 (FGF23) could be another potential mediator of the increased CV risk in patients with chronic hypoparathyroidism. FGF23 has been implicated as a CV risk factor, including for all-cause mortality, congestive heart failure, 
(a) Combined CV Endpoint

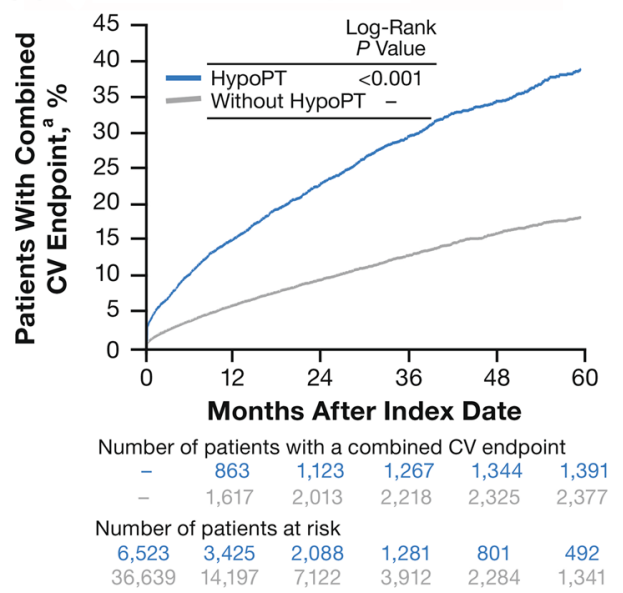

(b) $\mathrm{Ml}$

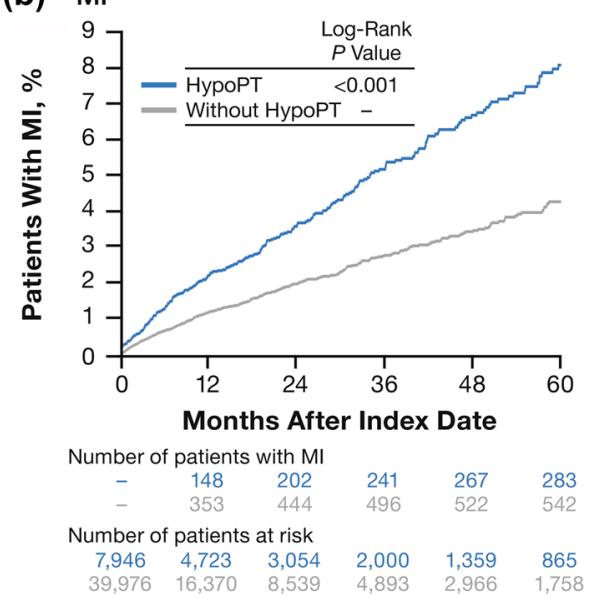

(c) Stroke

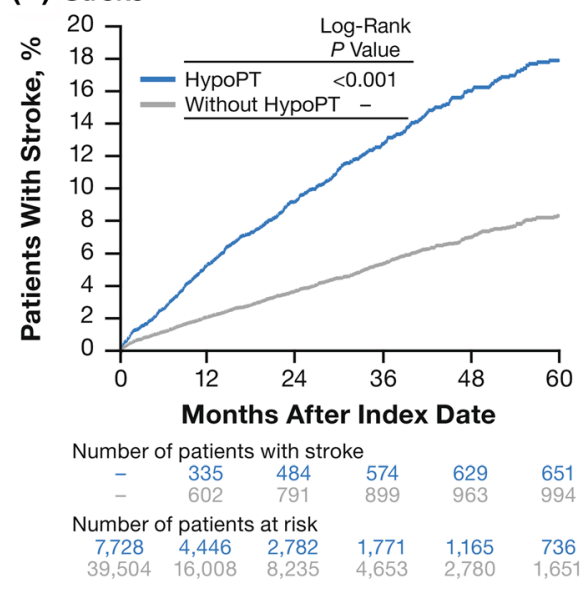

4Fig. 2 Time to first diagnosis during the study period among patients with no diagnoses of $\mathrm{CV}$ outcomes of interest during the baseline period for (a) combined CV endpoint $^{\mathrm{a}}$, (b) MI, and (c) stroke. $C V$ cardiovascular, HypoPT hypoparathyroidism, $M I$ myocardial infarction. ${ }^{a}$ Combined $\mathrm{CV}$ endpoint includes cerebrovascular disease, coronary artery disease, heart failure, and peripheral vascular disease

and left ventricular hypertrophy, in patients with CKD [28-30]. Additionally, elevated FGF23 levels were also linked to increased risk of death and CV disease in the general population [31]. FGF23 regulates kidney phosphate reabsorption [32], and higher FGF23 levels have been found in patients with higher serum phosphate concentrations [33]. Although FGF23 is not well studied in chronic hypoparathyroidism, FGF23 levels were found to be increased in a study including 12 patients with transient postsurgical hypoparathyroidism who also experienced hyperphosphatemia [34]. CV arrhythmias are likely to be associated with the course of the disease itself. The prolonged hypocalcemia that is characteristic of hypoparathyroidism has been found to increase QT interval [4, 7, 35-37], which can lead to arrhythmias [38]. However, FGF23-mediated left ventricular hypertrophy could also predispose patients to arrhythmias [39]. Because patients with chronic hypoparathyroidism are treated with conventional therapy, additional studies are needed to assess to what extent the observed risk is caused by hypoparathyroidism, its treatment with oral calcium and active vitamin $\mathrm{D}$, or both.

Our results should be evaluated in light of several limitations. As a result of the observational study design, it is possible that there are unobserved or unmeasured differences between cohorts that were not accounted for. Additionally, baseline characteristics between study groups were unbalanced, with individuals in the control group being younger and having lower prevalence of $\mathrm{CV}$ disease and $\mathrm{CV}$ risk factors. Nonetheless, the adverse association between chronic hypoparathyroidism and all individual and combined $\mathrm{CV}$ outcomes remained robust in the models adjusted for age, diabetes, 
Table 2 Risk of CV conditions in patients with chronic hypoparathyroidism compared with those without chronic hypoparathyroidism

\begin{tabular}{|c|c|c|c|}
\hline Outcome $^{a}$ & HR & $95 \% \mathrm{CI}$ & $P$ value \\
\hline \multicolumn{4}{|l|}{$\mathrm{AF}$} \\
\hline Unadjusted & 2.80 & $2.46-3.18$ & $<0.001$ \\
\hline Adjusted $^{\mathrm{b}}$ & 1.72 & $1.51-1.97$ & $<0.001$ \\
\hline \multicolumn{4}{|c|}{ Cerebrovascular disease } \\
\hline Unadjusted & 2.43 & $2.22-2.66$ & $<0.001$ \\
\hline Adjusted $^{\mathrm{b}}$ & 1.48 & $1.34-1.62$ & $<0.001$ \\
\hline \multicolumn{4}{|l|}{ CAD } \\
\hline Unadjusted & 2.21 & $2.01-2.43$ & $<0.001$ \\
\hline Adjusted ${ }^{\mathrm{b}}$ & 1.39 & $1.26-1.54$ & $<0.001$ \\
\hline \multicolumn{4}{|l|}{ MI } \\
\hline Unadjusted & 1.86 & $1.61-2.15$ & $<0.001$ \\
\hline Adjusted $^{\mathrm{b}}$ & 1.18 & $1.01-1.38$ & 0.036 \\
\hline \multicolumn{4}{|l|}{$\mathrm{HF}$} \\
\hline Unadjusted & 3.03 & $2.72-3.37$ & $<0.001$ \\
\hline Adjusted $^{\mathrm{b}}$ & 1.64 & $1.46-1.84$ & $<0.001$ \\
\hline \multicolumn{4}{|l|}{ PVD } \\
\hline Unadjusted & 2.77 & $2.54-3.03$ & $<0.001$ \\
\hline Adjusted $^{\mathrm{b}}$ & 1.66 & $1.51-1.81$ & $<0.001$ \\
\hline \multicolumn{4}{|l|}{ Stroke } \\
\hline Unadjusted & 2.40 & $2.18-2.66$ & $<0.001$ \\
\hline Adjusted $^{\mathrm{b}}$ & 1.45 & $1.31-1.62$ & $<0.001$ \\
\hline \multicolumn{4}{|c|}{ Tachyarrhythmia } \\
\hline Unadjusted & 2.52 & $2.01-3.15$ & $<0.001$ \\
\hline Adjusted $^{\mathrm{b}}$ & 1.68 & $1.32-2.14$ & $<0.001$ \\
\hline \multicolumn{4}{|c|}{ Combined CV endpoint $^{c}$} \\
\hline Unadjusted & 2.60 & $2.43-2.77$ & $<0.001$ \\
\hline Adjusted $^{\mathrm{b}}$ & 1.63 & $1.52-1.75$ & $<0.001$ \\
\hline
\end{tabular}

$A F$ atrial fibrillation, $C A D$ coronary artery disease, $C I$ confidence interval, $C K D$ chronic kidney disease, $C V$ cardiovascular, $H F$ heart failure, $H R$ hazard ratio, $M I$ myocardial infarction, $P V D$ peripheral vascular disease

a Patients without hypoparathyroidism served as the reference group for all analyses

b Multivariable Cox models adjusted for demographic (age, sex, race, region, and index year) and clinical (other comorbid CV conditions, $\mathrm{CKD}$, hyperlipidemia, hypertension, diabetes, and use of CV medications) characteristics at baseline

${ }^{c}$ Including cerebrovascular disease, CAD, HF, and PVD hypertension, CKD, underlying CV disease, and differences in baseline CV medications. Full medical histories were not available, limiting the ability to acquire information about important confounders, such as smoking history or family history of CV disease. We were unable to evaluate the use of calcium supplements, which are frequently obtained over the counter (and thus not visible in claims data), and no information about active vitamin $\mathrm{D}$ analogues was available. As with all retrospective claims database analyses, there could be miscoded and/or missing data. Additionally, this study was limited to the USA. We would not expect the conclusions to vary across different countries or regions; nonetheless, the magnitude of the results may differ in other settings because of different distributions of effect modifiers (e.g., different prevalence of comorbidities at baseline and different treatment practices).

Nevertheless, to our knowledge, the current investigation is the largest study to date that has evaluated the risk of developing various incident CV conditions among 8097 and 40,485 individuals with and without chronic hypoparathyroidism, respectively, from a nationally representative database. Our research contributes to the emerging concern over the potential risk of $\mathrm{CV}$ events among patients with chronic hypoparathyroidism.

\section{CONCLUSIONS}

In this retrospective study using a large database analysis, patients with chronic hypoparathyroidism had an increased risk of all individual $\mathrm{CV}$ outcomes and the composite CV outcome compared with patients without hypoparathyroidism after adjustment for potential confounders. This study contributes to emerging evidence concerning the link between hypoparathyroidism and $\mathrm{CV}$ outcomes and suggests a need for increased monitoring and awareness of $\mathrm{CV}$ risk in patients with chronic hypoparathyroidism. The mechanisms that mediate the relationship between chronic hypoparathyroidism and CV conditions warrant further research. 


\section{ACKNOWLEDGEMENTS}

Funding. This research and the journal's Rapid Service and Open Access fees were funded by Shire Human Genetic Therapies, Inc., a Takeda company, Lexington, MA, USA.

Authorship. All named authors meet the International Committee of Medical Journal Editors (ICMJE) criteria for authorship for this article, take responsibility for the integrity of the work as a whole, and have given their approval for this version to be published.

Author Contributions. KC, FM, ES, AB and NS contributed to the conception and design of the research. KC, FM, ES, and $\mathrm{AB}$ contributed to the acquisition and analysis of data. All authors (EOG, KC, MK, LR, FM, ES, AB, NS, and SK) contributed to the interpretation of the data, drafting of the manuscript, critical revision of the manuscript, agree to be fully accountable for ensuring the integrity and accuracy of the work, and read and approved the final manuscript.

Medical Writing and Editorial Assistance. Under the direction of the authors, writing and editorial support in the preparation of this manuscript was provided by Alan Storey, PhD, an employee of ICON (Marlow, Buckinghamshire, UK), and funded by Shire Human Genetic Therapies, Inc., a Takeda company, Lexington, MA, USA.

Disclosures. Elvira O. Gosmanova has served as a consultant for Shire, a Takeda company. Kristina Chen is a former employee of Shire Human Genetic Therapies, Inc., a Takeda company, Lexington, MA, USA; current affiliation is Arena Pharmaceuticals, Boston, MA, USA. Markus Ketteler has served as a research investigator and consultant for Shire, a Takeda company. Lars Rejnmark has served as a consultant and speaker for Shire, a Takeda company. Fan Mu, Elyse Swallow are employees, and Allison Briggs is a former employee of Analysis Group, Inc. contracted by Shire, a Takeda company, to conduct this research. Nicole Sherry is a former employee of Shire Human Genetic Therapies, Inc., a Takeda company, Lexington, MA, USA. Sanjiv Kaul has served as an advisory board member for Ultromics and as a consultant for Shire, a Takeda company. Elvira O. Gosmanova is an employee of the US Department of Veterans Affairs. Opinions expressed in this article are those of the authors and do not necessarily represent the opinion of the US Department of Veterans Affairs.

Compliance with Ethics Guidelines. Because this study used de-identified licensed data from a HIPAA-compliant managed care database, ethics committee approval and informed consent were not required.

Data Availability. The datasets generated and/or analyzed during the current study from the managed care claims database are not publicly available but are available from the corresponding author on reasonable request.

Prior Presentations. Initial data from this study were presented at the Endocrine Society Annual Meeting; March 23-26, 2019, New Orleans, LA, USA; and at the 21st European Congress of Endocrinology; May 18-21, 2019, Lyon, France.

Open Access. This article is licensed under a Creative Commons Attribution-NonCommercial 4.0 International License, which permits any non-commercial use, sharing, adaptation, distribution and reproduction in any medium or format, as long as you give appropriate credit to the original author(s) and the source, provide a link to the Creative Commons licence, and indicate if changes were made. The images or other third party material in this article are included in the article's Creative Commons licence, unless indicated otherwise in a credit line to the material. If material is not included in the article's Creative Commons licence and your intended use is not permitted by statutory regulation or exceeds the permitted use, you will need to obtain permission directly from the copyright holder. To view a copy of this licence, visit http://creativecommons.org/licenses/bync/4.0/. 


\section{REFERENCES}

1. Abate EG, Clarke BL. Review of hypoparathyroidism. Front Endocrinol (Lausanne). 2017;7:172.

2. Bilezikian JP, Khan A, Potts JT Jr, et al. Hypoparathyroidism in the adult: epidemiology, diagnosis, pathophysiology, target-organ involvement, treatment, and challenges for future research. J Bone Miner Res. 2011;26:2317-37.

3. Shoback DM, Bilezikian JP, Costa AG, et al. Presentation of hypoparathyroidism: etiologies and clinical features. J Clin Endocrinol Metab. 2016;101:2300-12.

4. Kudoh C, Tanaka S, Marusaki S, et al. Hypocalcemic cardiomyopathy in a patient with idiopathic hypoparathyroidism. Intern Med. 1992;31:561-8.

5. Shoback D. Hypoparathyroidism. N Engl J Med. 2008;359:391-403.

6. Tabacco G, Naciu AM, Maggi D, et al. Cardiovascular autonomic neuropathy as a new complication of postsurgical chronic hypoparathyroidism. J Bone Miner Res. 2019;34:475-81.

7. Underbjerg L, Sikjaer T, Rejnmark L. Long-term complications in patients with hypoparathyroidism evaluated by biochemical findings: a case-control study. J Bone Miner Res. 2018;33:822-31.

8. Cozzolino M, Brancaccio D, Gallieni M, Slatopolsky E. Pathogenesis of vascular calcification in chronic kidney disease. Kidney Int. 2005;68:429-36.

9. Mizobuchi M, Ogata H, Koiwa F, Kinugasa E, Akizawa T. Vitamin D and vascular calcification in chronic kidney disease. Bone. 2009;45(Suppl 1): S26-9.

10. Wang AY-M, Wang M, Woo J, et al. Cardiac valve calcification as an important predictor for all-cause mortality and cardiovascular mortality in long-term peritoneal dialysis patients: a prospective study. J Am Soc Nephrol: JASN. 2003;14:159-68.

11. Underbjerg L, Sikjaer T, Mosekilde L, Rejnmark L. Cardiovascular and renal complications to postsurgical hypoparathyroidism: a Danish nationwide controlled historic follow-up study. J Bone Miner Res. 2013;28:2277-85.

12. Underbjerg L, Sikjaer T, Mosekilde L, Rejnmark L. The epidemiology of nonsurgical hypoparathyroidism in Denmark: a nationwide case finding study. J Bone Miner Res. 2015;30:1738-44.

13. Underbjerg L, Sikjaer T, Rejnmark L. Cardiovascular findings in patients with nonsurgical hypoparathyroidism and pseudohypoparathyroidism: a cohort study. Clin Endocrinol (Oxf). 2019;90:592-600.

14. Vadiveloo T, Donnan PT, Leese CJ, Abraham KJ, Leese GP. Increased mortality and morbidity in patients with chronic hypoparathyroidism: a population-based study. Clin Endocrinol (Oxf). 2019;90:285-92.

15. Brandi ML, Bilezikian JP, Shoback D, et al. Management of hypoparathyroidism: summary statement and guidelines. J Clin Endocrinol Metab. 2016;101:2273-83.

16. Nandish S, Wyatt J, Bailon O, Smith M, Oliveros R, Chilton R. Implementing cardiovascular risk reduction in patients with cardiovascular disease and diabetes mellitus. Am J Cardiol. 2011;108:42B$51 \mathrm{~B}$.

17. Vadiveloo T, Donnan PT, Leese GP. A populationbased study of the epidemiology of chronic hypoparathyroidism. J Bone Miner Res. 2018;33: 478-85.

18. Bilezikian JP, Brandi ML, Cusano NE, et al. Management of hypoparathyroidism: present and future. J Clin Endocrinol Metab. 2016;101:2313-24.

19. Bollerslev J, Rejnmark L, Marcocci C, et al. European Society of Endocrinology clinical guideline: treatment of chronic hypoparathyroidism in adults. Eur J Endocrinol. 2015;173:G1-120.

20. Anderson JJ, Kruszka B, Delaney JA, et al. Calcium intake from diet and supplements and the risk of coronary artery calcification and its progression among older adults: 10-year follow-up of the MultiEthnic Study of Atherosclerosis (MESA). J Am Heart Assoc. 2016;5:e003815.

21. Li K, Kaaks R, Linseisen J, Rohrmann S. Associations of dietary calcium intake and calcium supplementation with myocardial infarction and stroke risk and overall cardiovascular mortality in the Heidelberg cohort of the European Prospective Investigation into Cancer and Nutrition study (EPICHeidelberg). Heart. 2012;98:920-5.

22. Tankeu AT, Ndip Agbor V, Noubiap JJ. Calcium supplementation and cardiovascular risk: a rising concern. J Clin Hypertens (Greenwich). 2017;19: 640-6.

23. Bolland MJ, Grey A, Reid IR. Calcium supplements and cardiovascular risk: 5 years on. Ther Adv Drug Saf. 2013;4:199-210.

24. Michaelsson $\mathrm{K}$, Melhus $\mathrm{H}$, Warensjo Lemming E, Wolk A, Byberg L. Long term calcium intake and rates of all cause and cardiovascular mortality: 
community based prospective longitudinal cohort study. BMJ. 2013;346:f228.

25. Ejlsmark-Svensson H, Rolighed L, Rejnmark L. Effect of parathyroidectomy on cardiovascular risk factors in primary hyperparathyroidism: a randomized clinical trial. J Clin Endocrinol Metab. 2019;104:3223-32.

26. Lorente-Poch L, Rifa-Terricabras S, Sancho JJ, Torselli-Valladares D, Gonzalez-Ortiz S, Sitges-Serra A. Prevalence of basal ganglia and carotid artery calcifications in patients with permanent hypoparathyroidism after total thyroidectomy. Endocr Connect. 2020;9:955-62.

27. Lewis JR, Radavelli-Bagatini S, Rejnmark L, et al. The effects of calcium supplementation on verified coronary heart disease hospitalization and death in postmenopausal women: a collaborative metaanalysis of randomized controlled trials. J Bone Miner Res. 2015;30:165-75.

28. Isakova T, Xie H, Yang W, et al. Fibroblast growth factor 23 and risks of mortality and end-stage renal disease in patients with chronic kidney disease. JAMA. 2011;305:2432-9.

29. Scialla JJ, Xie H, Rahman M, et al. Fibroblast growth factor-23 and cardiovascular events in CKD. J Am Soc Nephrol. 2014;25:349-60.

30. Gutierrez OM, Januzzi JL, Isakova T, et al. Fibroblast growth factor 23 and left ventricular hypertrophy in chronic kidney disease. Circulation. 2009;119: 2545-52.

31. Ix JH, Katz R, Kestenbaum BR, et al. Fibroblast growth factor- 23 and death, heart failure, and cardiovascular events in community-living individuals: CHS (Cardiovascular Health Study). J Am Coll Cardiol. 2012;60:200-7.
32. Liu S, Quarles LD. How fibroblast growth factor 23 works. J Am Soc Nephrol. 2007;18:1637-47.

33. Gutierrez OM, Wolf M, Taylor EN. Fibroblast growth factor 23, cardiovascular disease risk factors, and phosphorus intake in the health professionals follow-up study. Clin J Am Soc Nephrol. 2011;6: 2871-8.

34. Yamashita $H$, Yamazaki $Y$, Hasegawa $H$, et al. Fibroblast growth factor-23 (FGF23) in patients with transient hypoparathyroidism: its important role in serum phosphate regulation. Endocr J. 2007;54:465-70.

35. Bashour T, Basha HS, Cheng TO. Hypocalcemic cardiomyopathy. Chest. 1980;78:663-5.

36. Fisher NG, Armitage A, McGonigle RJ, Gilbert TJ. Hypocalcaemic cardiomyopathy; the relationship between myocardial damage, left ventricular function, calcium and ECG changes in a patient with idiopathic hypocalcaemia. Eur J Heart Fail. 2001;3: 373-6.

37. Meyer T, Ruppert V, Karatolios K, Maisch B. Hereditary long QT syndrome due to autoimmune hypoparathyroidism in autoimmune polyendocrinopathy-candidiasis-ectodermal dystrophy syndrome. J Electrocardiol. 2007;40:504-9.

38. Postema PG, Wilde AA. The measurement of the QT interval. Curr Cardiol Rev. 2014;10:287-94.

39. Mehta R, Cai X, Lee J, et al. Association of fibroblast growth factor 23 with atrial fibrillation in chronic kidney disease, from the chronic renal insufficiency cohort study. JAMA Cardiol. 2016;1:548-56. 